\title{
Review
}

\section{Novel Insights into Membrane Targeting of B Cell Lymphoma}

Charlotte M. de Winde, ${ }^{1}$ Suraya Elfrink, ${ }^{1}$ and Annemiek B. van Spriel ${ }^{1, *}$

Standard therapy of patients with B cell non-Hodgkin lymphoma (B-NHL) predominantly consists of chemotherapy combined with anti-CD20 (e.g., rituximab) immunotherapy. However, relapse of aggressive B-NHL occurs frequently, and this may coincide with therapy resistance. This demonstrates the urgent need for exploring new lymphoma-targeted therapies. We review here recent insights in the pathophysiology of $\mathrm{B}-\mathrm{NHL}$ and discuss CD20 and three alternative membrane targets (B cell receptor, immune checkpoints PD-1/ PD-L1, tetraspanin CD37) that are currently in the spotlight for B-NHL treatment. Furthermore, we present a novel concept in which the plasma membrane organization of the lymphoma B cell determines the efficacy of membranetargeted therapies, and this has consequences for treatment application and clinical outcome in patients with B cell lymphoma.

\section{Origin of B Cell Non-Hodgkin Lymphoma}

B cells undergo several distinct phases of development, starting from pre-B cells in the bone marrow towards ultimately their differentiation into antibody-producing plasma cells and memory B cells. Upon encountering specific antigens, germinal centers (GCs, see Glossary) are formed within the lymph node, where B cells undergo somatic hypermutation (SHM) and class-switch recombination (CSR) to generate a high-affinity B cell receptor (BCR). However, SHM and CSR are error-prone mechanisms, and hence the majority of B cell nonHodgkin lymphomas (B-NHL) arise from GC B cells [1]. These GC-derived B cell lymphomas frequently harbor chromosomal translocations of proto-oncogenes such as $B C L 2, B C L 6$, or MYC that are relocated under the control of the active immunoglobulin heavy chain ( $\mathrm{lgH}$ ) locus $[1,2]$. Because these translocations often involve the non-productively rearranged lg loci, most lymphomas still express a functional BCR [1].

The GC-derived B-NHLs include follicular lymphoma (FL), Burkitt lymphoma (BL), and diffuse large $\mathbf{B}$ cell lymphoma (DLBCL). DLBCL is the most prevalent type, accounting for approximately one third of all NHL cases. Two subtypes of DLBCL have been identified: germinal center $B$ cell-like (GCB-) DLBCL, arising from GC B cells, and activated B cell-like (ABC-) DLBCL, which has a post-GC origin [3]. ABC-DLBCL is characterized by constitutively active BCR/NF-KB signaling [4], and has a worse prognosis than GCB-DLBCL [3]. The hallmark of $B L$ is translocation of $M Y C$ to the IgH locus [5]. Both DLBCL and $B L$ are aggressive cancers, in contrast to FL which is an indolent lymphoma. However, in a large proportion of FL cases the disease transforms into the more aggressive DLBCL [6]. Current first-line treatment of DLBCL consists of the combination of rituximab (anti-CD20 monoclonal antibody, mAb) with CHOP-based chemotherapy, frequently followed by subsequent radiotherapy. BL is treated with $\mathrm{CHOP}$-based chemotherapy, sometimes combined with rituximab. For FL, a watch-andwait policy is frequently applied. In case of treatment indication, first-line treatment consists of
Trends

B cell lymphoma patients still face disease relapse after standard therapy (e. g., R-CHOP).

The cell surface of lymphoma B cells is the most easily accessible part of the cell, and therefore a favorable target.

In addition to CD20, alternative B cell membrane proteins, including the $B$ cell receptor and tetraspanin CD37, have gained renewed interest as therapeutic targets.

Immune checkpoint inhibitors currently tested in (pre)clinical studies for B cell lymphoma may stimulate anti-lymphoma immune responses.

The expression of membrane-organizing proteins, such as tetraspanins, differs on lymphoma B cells compared to healthy $B$ cells.
${ }^{1}$ Department of Tumor Immunology, Radboud Institute for Molecular Life Sciences, Radboud University Medical Center, Nijmegen, The Netherlands

${ }^{*}$ Correspondence:

Annemiek.vanSpriel@radboudumc.nl (A.B. van Spriel). 


\section{Trends in Cancer}

rituximab combined with CHOP- or CVP-based chemotherapy, with or without subsequent radiotherapy. The exact protocol depends on the diagnosis and condition of the patient [7-9].

Although immunotherapy with rituximab has significantly improved the clinical outcome of patients with B cell malignancies [10,11], many patients with DLBCL [12], BL [11], or FL [13] suffer from treatment failure or relapse. Several novel targeted therapies are currently under (pre)clinical investigation, including therapies targeting the tumor microenvironment (reviewed in [14]) and therapies targeting the lymphoma cells directly, either intracellular or at the membrane (reviewed in [15]). Targeting membrane proteins to treat cancer has been extensively studied by researchers and clinicians during the past two decades because the cell surface is the most accessible part of the cell. Rituximab is a clear example of successful antibody-based immunotherapy targeting a membrane protein. Antibody-based membranetargeted therapies act through different mechanisms, including direct cell death signaling, antibody-dependent cell-mediated cytotoxicity (ADCC) and complement-dependent cytotoxicity $(C D C)$. In this review we focus on recent developments and new membranetargeted therapies for GC-derived B cell lymphoma (Figure 1A). Furthermore, we propose a model illustrating the dynamic cell-surface protein landscape of lymphoma B cells that influences the efficacy of membrane-targeted therapies in B-NHL.

\section{CD20 as the First B Cell-Specific Membrane Target to Treat B-NHL}

Anti-CD20 mAb rituximab represents the first mAb approved for cancer therapy by the FDA in 1997. CD20 is a four-transmembrane protein expressed on mature $B$ cells and is involved in $B$ cell activation and differentiation, mainly by controlling calcium influx [16]. CD20 was selected in the early 1980s as new target for B-NHL because the majority of B-NHL cells (90\%) express a high density of CD2O at the cell surface [17]. Pro-B cells and antibody-producing plasma cells do not express CD20, and are therefore not vulnerable to CD20 targeting. As such, the healthy $\mathrm{B}$ cell pool is restored after rituximab treatment and B cell immune responses are largely preserved.

Anti-CD20 mAbs have been classified into types I and II [18], both of which induce ADCC. Type I mAbs (e.g., rituximab and ofatumumab) are regarded as the most potent antibodies because of their capacity to initiate CDC, in contrast to type II mAbs (e.g., obinutuzumab). Furthermore, type II mAbs may induce direct cell death (Figure 1B), although the exact mechanism remains to be elucidated. Upon type I mAb binding, CD20 is redistributed to specific microdomains, including lipid rafts and tetraspanin-enriched microdomains (Box 1), which stimulates clustering of the antibody Fc regions involved in CDC. Unfortunately, FC receptor polymorphisms and downregulation of CD20 caused by repeated exposure to rituximab [19] demonstrate the urgent need for development of alternative membrane-targeted strategies.

\section{Box 1. Plasma Membrane Microdomains}

The plasma membrane is composed of multiple different proteins and lipids that are non-randomly localized into specialized areas (microdomains). Signaling molecules, lipids, and proteins can cluster together in such organized domains, which is crucial for efficient signal transduction [73,74]. Disrupting membrane organization interferes with several membrane-proximal signaling processes, including those involved in tumor cell survival and metastasis (reviewed in [73]). Different types of membrane microdomains have been reported, including lipid rafts and tetraspanin-enriched microdomains (TEMs) [75]. Lipid rafts were originally identified as membrane fractions that are insoluble in the strong detergent Triton X-100 [76], hence they are also defined as detergent-resistant membrane domains (DRMs). They are enriched in densely packed sphingolipids and cholesterol, and harbor glycophosphatidylinositol (GPI)anchored proteins, among others [77]. A different type of microdomain is formed by tetraspanin proteins (Box 2) that associate with themselves and with specific partner molecules (including CD20, integrins, and MHCII) in clusters known as the tetraspanin web or TEMs [78-80]. Although both TEMs and lipid rafts are detergent-resistant and present in DRMs, they are very different domains in terms of protein composition $[81,82]$.

\section{Glossary}

Antibody-dependent cellmediated cytotoxicity (ADCC): the induction of cell death via binding of an antibody to its cell surface target. The Fc tail of the antibody engages Fc receptor-expressing immune cells, such as natural killer (NK) cells and macrophages, that kill the antibodybound target (i.e., tumor) cell. Burkitt lymphoma (BL): an aggressive type of $B$ cell nonHodgkin lymphoma characterized by deregulation of the MYC protooncogene.

CHOP: abbreviation for cyclophosphamide-doxorubicin (hydroxydaunorubicin)-vincristine (oncovin)-prednisone, a chemotherapeutic cocktail mostly used to treat $\mathrm{B}$ cell non-Hodgkin lymphoma patients. $\mathrm{CHOP}$ is often used in combination with anti-CD20 mAb rituximab (R-CHOP).

Class-switch recombination (CSR): the process by which the isotype or class of immunoglobulin produced by the $\mathrm{B}$ cell is switched, for example from IgM to lgG. This takes place via genomic changes in the locus encoding the constant region of the Ig heavy chain.

Complement-dependent cytotoxicity (CDC): the induction of cell death via activation of the complement system (C1q binding to the antibody), which leads to lysis of the target (i.e., tumor) cell membrane.

CVP: abbreviation for cyclophosphamide-vincristineprednisone, a chemotherapeutic cocktail used to treat follicular lymphoma patients.

Diffuse large B cell lymphoma (DLBCL): the most common and aggressive type of $B$ cell nonHodgkin lymphoma, accounting for approximately one third of all nonHodgkin lymphoma cases.

Follicular lymphoma (FL): the second most common B cell nonHodgkin lymphoma. FL is an indolent cancer, but can transform into the more aggressive DLBCL.

Germinal center: an area in B cell follicles of secondary lymphoid tissues where B cell proliferation, class-switch recombination, somatic hypermutation, and selection take place.

Somatic hypermutation (SHM): the process in which the Ig-encoding DNA of mature $B$ cells is subjected 


\section{Trends in Cancer}

(A)

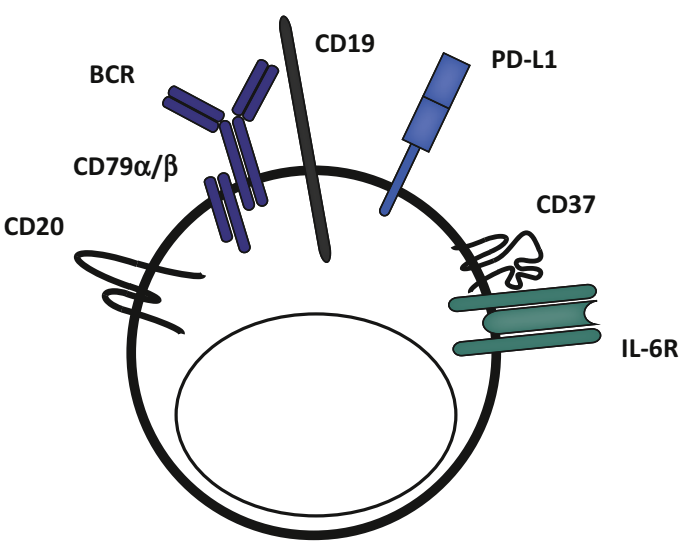

(B)

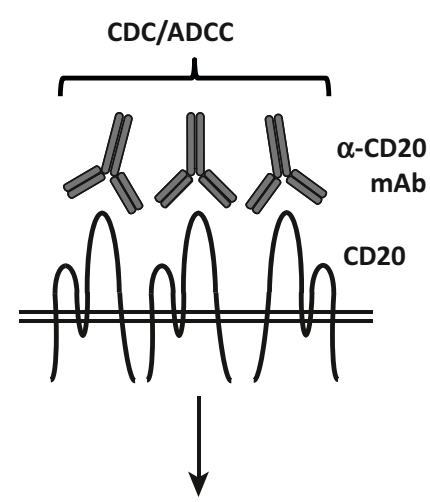

Apoptosis

(C) (i) Chronic active signaling

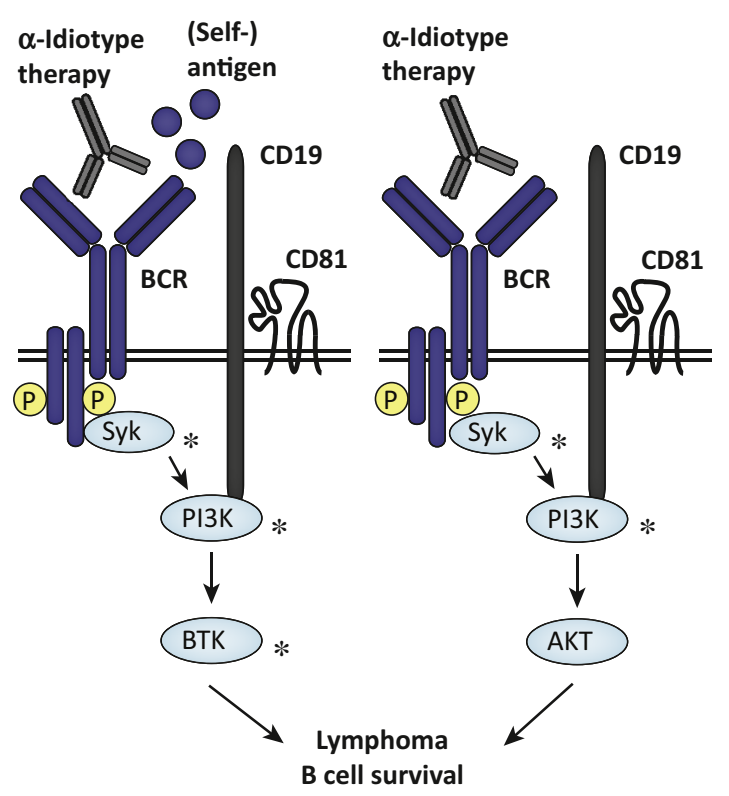

(D)

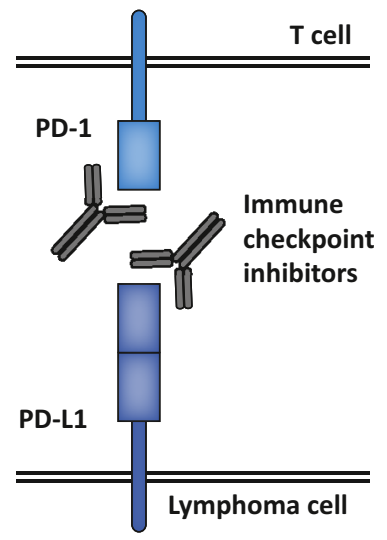

(E) (i) $\mathrm{CD} 37^{+}$lymphoma

(ii) $\mathrm{CD}^{-} 7^{-}$lymphoma

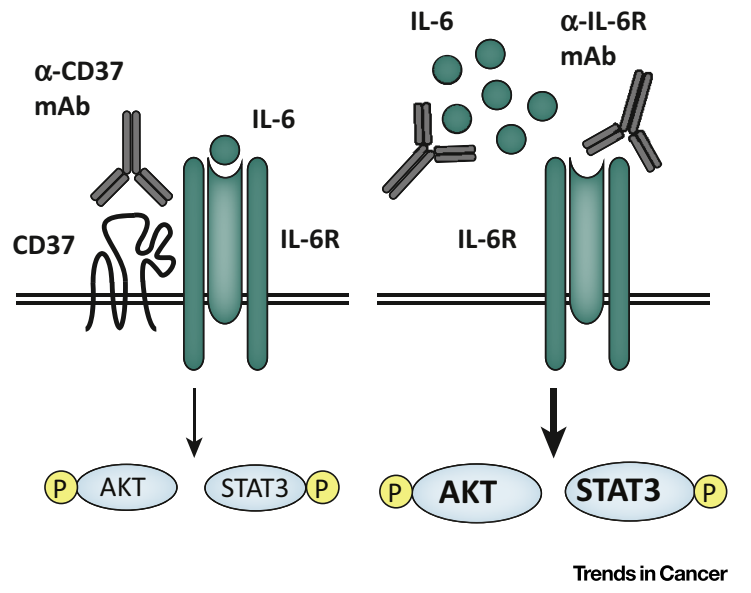

to the introduction of random point mutations. These modifications occur in the variable region of the $\lg$ gene leading to affinity maturation (increased-affinity BCR). 


\section{Trends in Cancer}

\section{Emerging Membrane-Targeted Therapies for B Cell Lymphoma}

In addition to CD20, numerous other potential membrane targets have been investigated in lymphoma, including CD19, CD22, CD23, CD37, CD47, CD52, CD74, CD79 $\alpha$, CD80, HLA$\mathrm{DR}$, and the BCR (idiotype). Furthermore, immune checkpoint inhibitors that target programmed cell death protein 1 (PD-1) and programmed cell death ligand 1 (PD-L1) are currently in the spotlight for treatment of B-NHL. Targeting of some of these cell-surface proteins with mAbs has shown promising therapeutic efficacy, but targeting others seems less suitable because of limited specificity (e.g., CD80 and HLA-DR are very broadly expressed) or resistance development (e.g., by antigen shedding or internalization). A few new mAbs directed against $B$ cell surface proteins currently tested in clinical trials show promising results either as first-line therapy in combination with rituximab or as monotherapy for heavily pretreated B-NHL patients (e.g., anti-CD22, epratuzumab; anti-CD37, otlertuzumab; anti-CD74, milatuzumab) (reviewed in [15]). Future antibody engineering studies, including conjugation to radio/immunotoxins or the generation of bispecific antibodies, may further improve their anti-lymphoma potency. Below we will discuss recent results and focus on novel developments in targeting membrane proteins in $\mathrm{B}-\mathrm{NHL}$, including immune checkpoints and tetraspanins (Figure 1).

\section{Aberrant BCR Function in B-NHL}

The BCR, a membrane-bound lg, can be divided into five classes (lgG, $\lg D, \lg M$, $\lg A$, or $\lg E$ ) that are important for antigen recognition and initiation of signaling events leading to $B$ cell survival, proliferation, and migration [20]. The BCR forms a complex with CD79 $\alpha$ (or Ig $\alpha$ ) and CD79 (or $\lg \beta$ ) which both contain an immunoreceptor tyrosine-based activation motif (ITAM) to transmit intracellular signaling. Upon antigen binding, the conformational state of the BCR changes to initiate downstream signaling, but the underlying molecular mechanisms are still debated. The 'conformation-induced oligomerization model', which proposes that single BCRs oligomerize upon antigen recognition [21], has recently been challenged by super-resolution studies. This has led to convincing evidence for the 'dissociation-activation model' in which BCR oligomers dissociate upon antigen binding, gaining an open conformation [22,23]. This conformational change influences the distribution of BCR coreceptors CD19 and CD20 that interact with IgD oligomers on resting B cells, and relocate to IgM oligomers upon B cell activation [23]. These changes in plasma membrane organization are controlled by a superfamily of membraneorganizing proteins, the tetraspanins (Box 2). Tetraspanin CD81 is required for reorganization of CD19 to BCR nanoclusters, and this enables binding of signaling proteins to the intracellular domain of CD19 and initiation of signaling downstream of the BCR [24,25].

Two modes of BCR signaling have been described. (i) Active signaling upon antigen binding to the BCR, leading to NF-kB activation via Syk, phosphoinositide 3-kinase (PI3K), and Bruton's tyrosine kinase (BTK); and (ii) tonic signaling, an antigen-independent mechanism required for $\mathrm{B}$

Figure 1. Current and Novel Membrane-Targeted Therapies in B Cell Lymphoma. (A) Membrane proteins used in and explored for lymphoma-targeted therapy. Left to right: CD20; B cell receptor (BCR) with its signaling proteins $C D 79 \alpha / \beta$; CD19; PD-L1; tetraspanin CD37 with the IL-6R. (B) Targeting CD20 using mAb (e.g., rituximab) will cluster CD20 proteins leading to tumor cell death via CDC, ADCC and/or direct apoptosis. (C) In chronic active BCR signaling (i), binding of (self) antigens to the BCR triggers recruitment of Syk to the phosphorylated (P) ITAMs of CD79 $\alpha / \beta$, which leads to translocation of PI3K to CD19. Subsequently, BTK is activated which initiates NF-KB activation via the CARMA1-BCL10-MALT1 complex resulting in lymphoma B cell survival. In tonic BCR signaling (ii), constitutive activation of PI3K in absence of (self) antigens results in continuous signaling via the AKT pathway and lymphoma cell survival. Novel anti-idiotype therapy strategies can directly target the BCR. Syk, PI3K, and BTK can be inhibited using small-molecule inhibitors (*). (D) Immune checkpoint inhibitors (e.g., nivolumab, pembrolizumab, pidilimumab) bind to PD-L1 expressed on lymphoma B cells or to PD-1 on T cells. This inhibits the immune-escape mechanism used by ABC-DLBCL and FL cells. (E) In CD37-expressing $\left(\mathrm{CD} 37^{+}\right) \mathrm{B}$ cell lymphoma (i), tumor cell death can be induced by targeting CD37-specific mAbs (IMGN529, Otlertuzumab). By contrast, CD37-negative (CD37 ${ }^{-}$) B cell lymphoma ( $50 \%$ of DLBCL cases) (ii), shows constitutive activation of the IL-6 signaling pathway (increased $\mathrm{p}-\mathrm{AKT}$ and $\mathrm{p}-\mathrm{STAT} 3$ ). These patients may benefit from $\alpha-\mathrm{IL}-6(\mathrm{R})$ therapy. Abbreviations: $A B C$, activated B cell-like; $A D C C$, antibody-dependent cell-mediated cytotoxicity; $C D C$, complement-dependent cytotoxicity; DLBCL, diffuse large B cell lymphoma; ITAM, immunoreceptor tyrosine-based activation motif. 


\title{
Trends in Cancer
}

\begin{abstract}
Box 2. Tetraspanins in Cancer Development
Tetraspanins are a family of four-transmembrane proteins involved in plasma membrane organization $[79,82]$. The mammalian tetraspanin superfamily consists of 33 members which are expressed on almost all cells and tissues [83]. Tetraspanins consist of four transmembrane domains, a short extracellular loop (EC1), a large extracellular loop (EC2) containing a conserved CCG region, a small intracellular loop, and two short intracellular tails [84,85]. Despite its similar structure, CD20 cannot be classified as a genuine tetraspanin protein because it lacks the conserved CCG region in the large extracellular loop [86]. The EC2 and transmembrane domains are involved in the formation of tetraspanin-enriched microdomains (TEMs) by lateral associations with interaction partners, including other tetraspanins and integrins [78-80]. Their short intracellular tails can interact with cytosolic signaling molecules (e.g., PKC, Pi4K, Rac $[63,79,85])$. Through their multiple interaction partners, tetraspanins are involved in different cellular processes including survival, proliferation, adhesion, and migration [78]. Recently, several studies have shown that tetraspanins contribute to cancer development and metastasis, and tetraspanin expression has been associated with patient outcome [56,87]. Therefore, tetraspanins are interesting targets for cancer therapy, and different (pre)clinical studies using tetraspanin targeting strategies are currently ongoing (https://clinicaltrials.gov) [61,87].
\end{abstract}

cell survival which occurs in mature resting $B$ cells via activation of PI3K and protein kinase $B$ (AKT) (reviewed in [26]) (Figure 1C). In most GC-derived B cell lymphomas, BCR signaling is disturbed, resulting in enhanced B cell survival and proliferation. BL shows tonic BCR signaling (Figure $1 \mathrm{C}$ ) via constitutive activation of the PI3K pathway driven by MYC translocation to the IgH locus during SHM $[5,27]$. Chronic active BCR signaling is seen in ABC-DLBCL, as evidenced by knockdown of BTK or components of the BCR complex that induces killing of lymphoma cells [4]. By contrast, GCB-DLBCL cells were not killed, indicating they do not require $B C R$ signaling to survive. In the majority of $A B C$-DLBCL cases, self-antigens present in the tumor microenvironment may drive continuous BCR stimulation (Figure 1C) [28]. The presence of BCR clusters on ABC-DLBCL cells resembles BCR clusters observed on normal $B$ cells following antigen stimulation [4]. In 10\% of ABC-DLBCL cases, gain-of-function mutations in CARMA1 (also known as CARD11) were found to result in continuous NF-кB activation [29]. Furthermore, in $20 \%$ of ABC-DLBCL cases, somatic mutations in the CD79 $\alpha /$ CD79 $\beta$ ITAM domains induced chronic active BCR signaling via inhibition of BCR endocytosis and prevention of binding of Lyn to CD79 $\beta$ [4].

In FL, constitutive BCR signaling induced by presence of (self-)antigens has been found in only $20-25 \%$ of the cases $[30,31]$, whereas the majority of FL cases $(80 \%)$ bear somatic mutations in the IgM-BCR leading to increased incorporation of $\mathrm{N}$-glycosylation sites [32,33]. If this occurs in the antigen-binding side of the BCR it may prevent binding of (self-)antigens, but in turn can be recognized and stimulated by lectin receptors on myeloid cells in the tumor microenvironment $[34,35]$. This results in reorganization of the BCR complex (including CD19) into signaling platforms and persistent antigen-independent signaling via Syk and BTK.

Currently, different small-molecule inhibitors targeting signaling proteins downstream of the BCR (Syk, Lyn, PI3K, BTK) are under investigation as a novel therapy for B-NHL (reviewed in [26]). Nevertheless, increased understanding of the conformational changes taking place in the BCR upon antigen binding may open new opportunities to directly target the BCR itself. This idea was originally explored in the 1980s when treatment of lymphoma patients with antiidiotype antibodies (targeting the variable region of the BCR) revealed promising clinical results [36]. However, this approach required the generation of patient-specific idiotype antibodies, hampering clinical implementation. Recently, a new tool has been developed to screen and target tumor-specific idiotypes with small peptides linked to a pre-made IgG-Fc protein, socalled anti-idiotype 'peptibodies' [37], which directly induce cell death via BCR signaling and ADCC. Furthermore, the anti-CD79 $\beta$ antibody-drug conjugate polatuzumab vedotin may be promising for the treatment of $\mathrm{B}-\mathrm{NHL}$ patients [38], and is currently being investigated in clinical trials (https://clinicaltrials.gov). 


\section{Trends in Cancer}

Therapeutic strategies targeting the BCR coreceptor CD19 are encouraging for patients with relapsed/refractory B-NHL. CD19 chimeric-antigen receptors expressed by T cells (CD19-CAR T cells) consist of an extracellular domain specific for CD19 on B-NHL cells and intracellular domains to provide T cell stimulatory signals (e.g., CD3 $\zeta$ and CD28). Adoptive transfer of CD19CAR T cells has given complete responses in 50-60\% of B-NHL patients in Phase I/II clinical trials [39]. Furthermore, blinatumomab, a CD19/CD3 bispecific T cell engager (BiTE) generated from the antigen-binding regions of the single-chain antibodies of CD19 and CD3, is approved by the FDA for treatment of acute lymphoblastic leukemia (ALL), and clinical results are currently being evaluated for the treatment of other B cell malignancies including B-NHL [40]. Future research will be necessary to determine the consequences of targeting the BCR complex in $\mathrm{B}$ $\mathrm{NHL}$ patients, and in particular how this changes BCR conformation and downstream signaling.

\section{Function and Targeting of Immune Checkpoints PD-1/PD-L1 in B-NHL}

Cancer immunotherapy was chosen as the scientific breakthrough of 2013 by Science journal [41] in view of promising results with immune checkpoint inhibitors. Immune checkpoints are proteins that regulate immune cell activation to maintain self-tolerance and prevent autoimmunity (reviewed in [42]). More specifically, these checkpoints play an important role in controlling T cell priming and activation. For example, PD-1 on T cells transfers an inhibitory signal when engaged by PD-L1 expressed by tumor cells or activated T cells.

Tumor cells may exploit these checkpoints to escape or suppress the immune system by (over) expressing PD-L1. Currently, anti-PD-1 mAbs (pembrolizumab and nivolumab) and anti-PD-L1 mAbs (atezolizumab and durvalumab) demonstrate promising clinical results in the treatment of different solid tumors, including metastatic melanoma, urothelial carcinoma, and advanced non-small cell lung carcinoma (reviewed in [43]). This treatment strategy has also gained more attention in the B cell lymphoma field (Figure 1D) (reviewed in [42]), and nivolumab has recently been approved by the FDA for the treatment of relapsed/refractory Hodgkin lymphoma in view of promising clinical results [44].

For DLBCL, promising clinical results have been obtained with the anti-PD-1 mAbs pidilizumab and nivolumab $[45,46]$, and both anti-PD-1 and anti-PD-L1 mAbs are currently being tested in clinical trials (https://clinicaltrials.gov/). It has been shown that a subset of ABC-DLBCL tumors $(11-31 \%)$ displays high expression of PD-L1, in contrast to GCB-DLBCL tumors that virtually lack expression of PD-L1. This high PD-L1 expression has been suggested to underlie the poorer prognosis of ABC-DLBCL patients compared to patients with GCB-DLBCL $[47,48]$. Because PD-L1 expression is restricted to a minority of DLBCL patients, it is likely that only this group will benefit from immune checkpoint blockade $[48,49]$.

Although PD-L1 is rarely expressed in FL cells [42,47], FL patients may still benefit from immune checkpoint blockade because inhibition of PD-1-expressing tumor-infiltrating T cells by PD-L1expressing non-tumor cells in the tumor microenvironment can be reversed [50]. This has been supported by clinical studies demonstrating good responses in FL patients treated with antiPD-1 mAbs (nivolumab and pidilizumab) [46,51]. Trials with pembrolizumab in FL patients are currently ongoing (https://clinicaltrials.gov/). Several studies have reported that BL cells do not express PD-L1 [47,49], and PD-1/PD-L1 checkpoint inhibitors have therefore not yet been studied in BL. Nonetheless, the promising results of immune checkpoint inhibition in FL, which rarely expresses PD-L1, may be translated to BL. Taken together, targeting the immune checkpoint membrane proteins PD-1/PD-L1 seems to be a promising novel treatment option for a selected group of ABC-DLBCL and FL patients. However, it is still debated whether expression of PD-1/PD-L1 is of prognostic value for immune checkpoint inhibition therapy in B$\mathrm{NHL}[52,53]$. 


\title{
Trends in Cancer
}

\author{
CD37 Rediscovered as a B Cell Membrane Target in the Treatment of B- \\ $\mathrm{NHL}$
}

Tetraspanin CD37 recently regained attention as promising membrane target for mature B cell malignancies (Box 2). CD37 expression is restricted to the immune system, with highest abundance on mature B cells, and is absent in earlier stages of B cell development and decreased on plasma cells $[54,55]$. This pattern is also reflected in different $B$ cell lymphomas: CD37 is mostly expressed on B cell malignancies derived from mature B cells (although 50\% of DLBCL patients lack CD37 [56,57], as discussed below), but not in acute lymphoblastic lymphoma and multiple myeloma [58]. Comparable to CD20, this distinct expression pattern makes CD37 an interesting target in GC-derived B cell lymphomas. Already in 1989 the first CD37-targeting antibody (labeled with the radioactive isotope iodine 131) was tested in 10 refractory NHL patients, with promising clinical results [59]. However, because anti-CD20 treatment (rituximab) was introduced at the same time, anti-CD37 therapy was forgotten for almost two decades until the development of a novel CD37-targeting mAb-derived polypeptide re-established interest in this target [60].

Several different antibody-based CD37-targeting approaches are currently under investigation in Phase I and II trials for chronic lymphocytic leukemia (CLL) and refractory or relapsed NHL patients (https://clinicaltrials.gov; reviewed in [61]) (Figure 1E). Otlertuzumab (TRU-016), a humanized, antibody-derived CD37-targeting peptide, is closest to general clinical application in B cell malignancies. In a randomized Phase II study, relapsed CLL patients treated with the chemotherapeutic bendamustine plus otlertuzumab showed increased progression-free survival compared to patients treated with bendamustine alone [62].

The antitumor mechanisms by which anti-CD37 agents act include ADCC, CDC, and direct apoptosis signaling $[60,63,64]$. In addition, CD37-antibody complexes are known to be internalized, and this has led to the generation of novel anti-CD37 agents coupled to toxins (IMGN529, an anti-CD37 antibody conjugated to an anti-microtubule agent) [64] or radioactive labels ( ${ }^{177}$ Lu-tetulomab) [65]. Both agents showed promising results in NHL xenograft mouse models $[64,65]$. Moreover, ${ }^{177}$ Lu-tetulomab was internalized with a higher efficiency than ${ }^{177}$ Lu-rituximab complexes in vitro [65], and IMGN529 showed increased activity compared to rituximab or CVP chemotherapy in a FL-derived xenograft mouse model [64]. A Phase I clinical trial completed in July 2016 studied IMGN529 in relapsed/refractory NHL and CLL patients (NCT01534715), and several Phase I/II clinical studies to test ${ }^{177}$ Lu-tetulomab (Betalutin) are currently including NHL patients (https://clinicaltrials.gov).

Furthermore, new insights into the function of CD37 have prompted interest in combination strategies. CD37 is important for the survival of lgG1-secreting plasma cells through the membrane organization of $\alpha 4 \beta 1$ integrins and subsequent activation of the AKT signaling pathway [66]. The intracellular tails of CD37 can be tyrosine phosphorylated, leading to the initiation of apoptotic signaling via the $\mathrm{N}$-terminal immunoreceptor tyrosine-based inhibition motif (ITIM)-like domain, and opposing survival signaling via its C-terminal ITAM domain [63]. Both pathways are induced by the CD37-targeting mAb-derived polypeptide SMIP-016 which was the basis for the development of TRU-016. Although signaling to apoptosis is the most prominent, the efficiency of CD37 targeting could be improved using combination therapy with pro-survival PI3K inhibitors [63].

Importantly, recent studies revealed that $\sim 50 \%$ of DLBCL tumors are negative for CD37 expression, and its loss is a potential risk factor for $\mathrm{R}-\mathrm{CHOP}$ resistance and poor survival rates independent of the International Prognostic Index (IPI) $[56,57]$. The underlying mechanism involves enhanced activation of the IL-6 signaling pathway in CD37-negative lymphomas [56]. IL-6 is known to be involved in the development of many cancers, including hematological 


\section{Trends in Cancer}
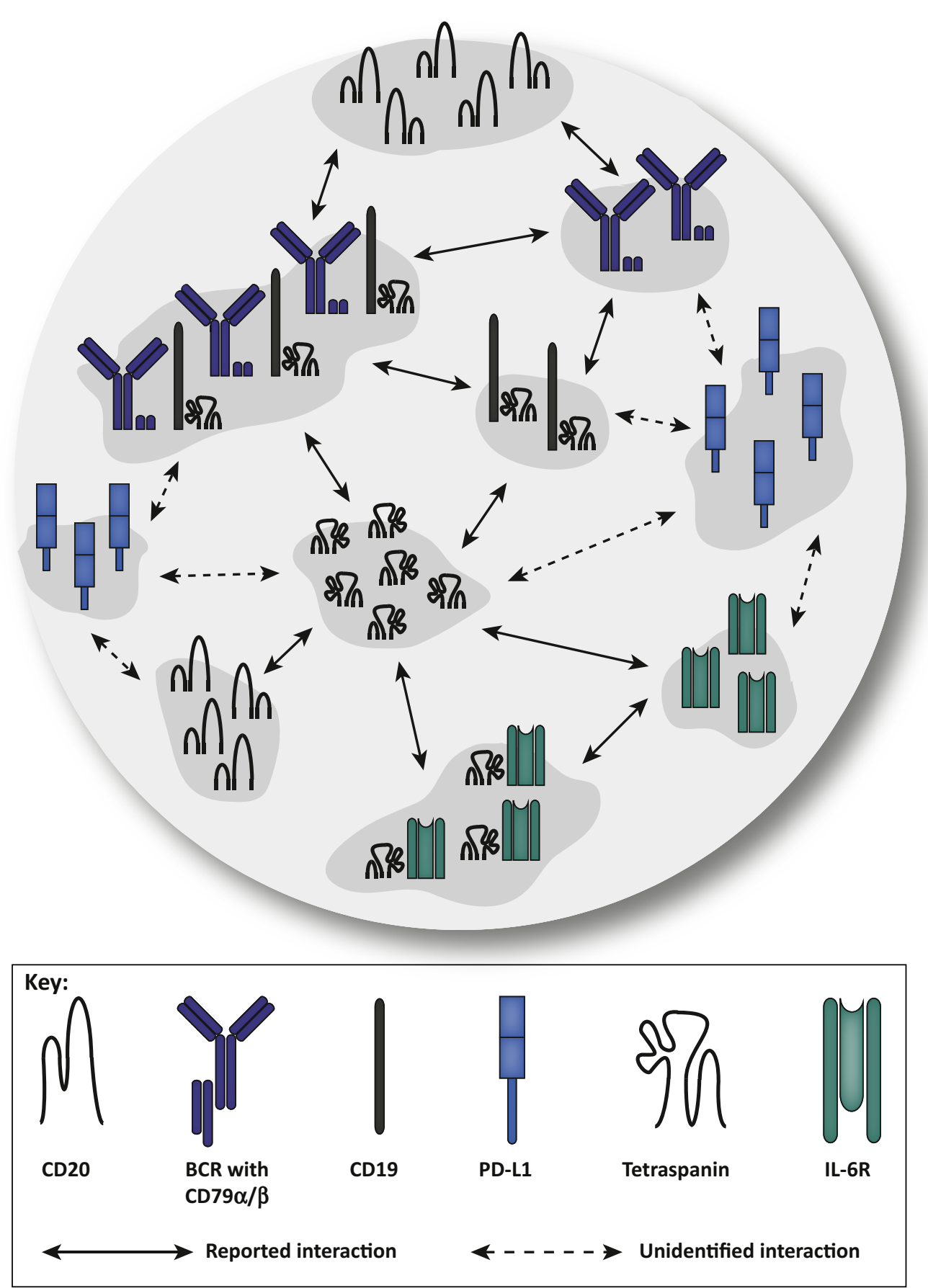

Trends in Cancer

Figure 2. Plasma Membrane Organization of Lymphoma B cells Determines the Efficacy of Membrane-Targeted Therapies. The cell membrane (light area) is highly organized into specialized microdomains (dark area) that are formed by dynamic homotypic (e.g., CD20-CD20) and heterotypic (e.g., CD19-CD81) protein-protein interactions. Upon ligand-receptor binding (e.g., IL-6 to IL-6R), induction of a conformational change can lead to rearrangement of protein-protein interactions and reorganization of the membrane landscape. Lymphoma B cells have a disrupted membrane protein organization, for example as a result of constant activation of the B cell receptor (BCR) by (self-)antigens, overexpression of PD-L1, or loss of tetraspanin CD37. In addition, BCR activation results in CD20 dissociation and redistribution, and anti-CD20 targeted therapy using rituximab induces CD20 clustering. These data support a model in which the microdomain organization of lymphoma cells determines the efficacy of membrane-targeted therapies, which has important implications for treatment application and clinical outcome. 


\section{Trends in Cancer}

malignancies, and several IL-6-targeting strategies have been developed (reviewed in $[67,68])$. Based on these studies, inhibition of IL-6 signaling (using mAb anti-IL-6 siltuximab or anti-IL-6R tocilizumab) may represent a potential new treatment strategy for patients with CD37-negative DLBCL (Figure 1E).

\section{Dynamic Protein Interactions Shape the Organization of the B Cell Membrane}

Proteins in the plasma membrane are not randomly distributed, and instead are localized to specific microdomains (Box 1). One of the first models of membrane protein organization on the B cell membrane was published two decades ago [69]. Using flow cytometry energy transfer (FCET) it was shown that CD20 interacts with tetraspanins CD53, CD81, and CD82 on a Iymphoma B cell line. In addition, confocal microscopy [70] and coimmunoprecipitation [71] revealed that $\mathrm{CD} 20$ also clusters with the $\mathrm{BCR}$ in specialized microdomains, indicating that they are localized in lipid rafts or tetraspanin microdomains (Box 1). The BCR dissociates from CD20 to distinct lipid rafts upon stimulation, and is subsequently internalized [70]. A direct interaction between CD20 and the BCR was recently confirmed using proximity-ligation assay [23]. It was shown that CD20 (similar to CD19) interacts with different BCR classes (IgD/lgM) on resting and antigen-activated human B cells, demonstrating redistribution of CD20 upon BCR stimulation.

CD20 has been shown to interact with several tetraspanins (CD53, CD81, and CD82) [69], although CD37 was not studied. Recently we discovered that both protein and mRNA expression of CD20 and CD37 on lymphoma B cells are correlated [57]. Although this is in line with the inferior outcome of CD37-negative DLBCL patients upon R-CHOP therapy, the prognostic significance of CD37 seems to be independent of CD20 mRNA levels [57]. Further research is necessary to verify a possible direct interaction and co-expression patterns of CD37 and CD2O in B-NHL cases.

These studies indicate that the organization of the B cell membrane is shaped by dynamic protein-protein interactions that are subject to change upon $\mathrm{B}$ cell activation. This protein organization is not only important for B cell function but also for the efficacy of membranetargeted therapies, as illustrated by clustering of CD20 upon rituximab binding [18]. Likewise, CD37-targeting using SMIP-016 resulted in translocation into microdomains, and translocation was required for efficient tumor cell apoptosis [63]. On lymphoma B cells, changes in membrane protein expression (e.g., absence of CD37) or protein clustering (e.g., conformational changes of the BCR upon binding of self-antigens) will change the protein organization and interactions at the plasma membrane. Based on these studies we propose that the changed cell-surface protein landscape of lymphoma B cells has consequences for the efficacy and application of membrane-targeted therapies (Figure 2). Moreover, targeted therapies may alter dynamic protein-protein interactions, affecting plasma membrane organization and downstream signaling.

\section{Concluding Remarks}

Many patients with B-NHL still face treatment failure or relapse upon standard therapy, emphasizing the urgent need for new treatment options. The plasma membrane is the most easily accessible part of the tumor cell, and exposes a variety of different proteins which may serve as treatment targets. We have discussed the widely studied target CD20 and three alternative membrane targets (BCR, PD-L1, and CD37) that are currently under investigation in clinical trials to treat B-NHL. Furthermore, patients with CD37-negative lymphoma B cells have a dismal prognosis and may benefit from anti-IL-6(R)-targeted therapy.

\section{Outstanding Questions}

What is the effect of membrane-targeted therapies on the plasma membrane organization of lymphoma cells? How does this alter signal transduction pathways and lymphoma cell death, and what is the effect on clinical outcome?

Is it possible to modify plasma membrane organization by targeting the tetraspanin web, and thereby induce downstream signaling that drives lymphoma cell eradication?

What is the effect of BCR-targeted therapies on the conformational state of the BCR, and on the BCR complex formation with partner proteins, including CD19, CD20, and CD81?

What is the predictive value of PD-L1 expression upon treatment with immune checkpoint inhibitors as measured by clinically relevant outcome parameters such as overall survival and progression-free survival?

Will Burkitt lymphoma patients benefit from immune checkpoint inhibitors given the lack of PD-L1 on these lymphoma cells? PD-L1-based therapy may target immune cells in the tumor microenvironment, and thereby eliminate the tumor-induced immune suppression.

One half of patients with DLBCL have lost CD37 expression on the tumor cells. Is CD37 deficiency also apparent in other B cell malignancies (e.g., FL, $\mathrm{BL}, \mathrm{CLL}$ ) and of prognostic value? This may have implications for inclusion criteria, stratification, and outcome of clinical trials studying CD37-targeted therapies.

Is targeting of IL-6, IL-6R, or downstream signaling proteins (JAK, STAT3) effective in patients with CD37-negative DLBCL that shows increased activation of the IL-6 pathway?

Which (membrane-targeting) combination therapies would be most effective to treat $\mathrm{B}$ cell lymphoma? 


\section{Trends in Cancer}

Membrane proteins interact with each other and thereby facilitate various cell biological processes, including initiation of downstream signaling. B cell activation will affect these protein interactions through induction of new interactions, more clustering, or alternatively by preventing specific interactions. Evidence is accumulating that lymphoma B cells contain aberrant cellsurface protein expression and organization, and this has important consequences for both the application and outcome of membrane-targeted therapy for B-NHL patients.

We are only at the beginning of understanding the membrane organization of normal and malignant B cells, and further research will be necessary to address the outstanding issues in this new and exciting field (see Outstanding Questions). In particular, recent advances in superresolution microscopy now enable imaging the membrane composition of lymphoma cells at the nanoscale level [72]. Taking into account that lymphomas are heterogeneous tumors [2], targeting membrane proteins as monotherapy will probably not be sufficient for complete tumor eradication. Thus, the use of combination therapies, such as immuno-chemotherapy and the additional use of small-molecule inhibitors, will be important in the future treatment of B cell non-Hodgkin lymphoma.

\section{Acknowledgments}

We would like to thank Blanca Scheijen and Dick-Johan van Spronsen (Radboud University Medical Center) for critically reading this manuscript. A.B.v.S. is recipient of a grant from The Netherlands Organization for Scientific Research (NWOALW VIDI grant 864.11.006), a Dutch Cancer Society grant (KUN2014-6845), and a European Research Council Consolidator Grant (Secret Surface, 724281).

\section{References}

1. Küppers, R. and Dalla-Favera, R. (2001) Mechanisms of chromosomal translocations in B cell lymphomas. Oncogene 20, 5580-5594

2. Blombery, P.A. et al. (2015) The molecular pathogenesis of B-cell non-Hodgkin lymphoma. Eur. J. Haematol. 95, 280-293

3. Alizadeh, A.A. et al. (2000) Distinct types of diffuse large B-cell lymphoma identified by gene expression profiling. Nature 403 , 503-511

4. Davis, R.E. et al. (2010) Chronic active B-cell-receptor signalling in diffuse large B-cell lymphoma. Nature 463, 88-92

5. Schmitz, R. et al. (2014) Oncogenic mechanisms in Burkitt lymphoma. Cold Spring Harb. Perspect. Med. 4, a014282

6. Kridel, R. et al. (2012) Pathogenesis of follicular lymphoma. J. Clin. Invest. 122, 3424-3431

7. Tilly, H. et al. (2015) Diffuse large B-cell lymphoma (DLBCL): ESMO Clinical Practice Guidelines for diagnosis, treatment and follow-up. Ann. Oncol. 26, v116-v125

8. Casulo, C. et al. (2015) Early relapse of follicular lymphoma after rituximab plus cyclophosphamide, doxorubicin, vincristine, and prednisone defines patients at high risk for death: an analysis from the National LymphoCare Study. J. Clin. Oncol. 33, 2516-2522

9. Dreyling, M. et al. (2016) Newly diagnosed and relapsed follicular Iymphoma: ESMO Clinical Practice Guidelines for diagnosis, treatment and follow-up. Ann. Oncol. 27, v83-v90

10. Sant, M. et al. (2014) Survival for haematological malignancies in Europe between 1997 and 2008 by region and age: results of EUROCARE-5, a population-based study. Lancet Oncol. 15, 931-942

11. Wildes, T.M. et al. (2014) Rituximab is associated with improved survival in Burkitt lymphoma: a retrospective analysis from two US academic medical centers. Ther. Adv. Hematol. 5, 3-12

12. Colosia, A. et al. (2014) Clinical efficacy and safety in relapsed/ refractory diffuse large B-cell lymphoma: a systematic literature review. Clin. Lymphoma Myeloma Leuk. 14, 343-355

13. Tan, D. et al. (2013) Improvements in observed and relative survival in follicular grade 1-2 lymphoma during 4 decades: the Stanford University experience. Blood 122, 981-987
14. Martin, M. et al. (2016) Targeting microenvironment in cancer therapeutics. Oncotarget 7, 52575-52583

15. Horn, H. et al. (2017) New targeted therapies for malignant lymphoma based on molecular heterogeneity. Expert Rev. Hematol. 10, 39-51

16. Tedder, T.E. and Engel, P. (1994) CD20: a regulator of cell-cycle progression of B lymphocytes. Immunol. Today 15, 450-454

17. Nadler, L. et al. (1981) A unique cell surface antigen identifying lymphoid malignancies of B cell origin. J. Clin. Invest. 67, 134-140

18. Beers, S.A. et al. (2010) CD20 as a target for therapeutic type i and ii monoclonal antibodies. Semin. Hematol 47, 107-114

19. Teo, E. et al. (2016) A review of monoclonal antibody therapies in lymphoma. Crit. Rev. Oncol. Hematol. 97, 72-84

20. Venkitaraman, A. et al. (1991) The B-cell antigen receptor of the five immunoglobulin classes. Nature 352, 777-781

21. Tolar, P. and Pierce, S. (2010) A conformation-induced oligomerization model for B cell receptor microclustering and signaling. Curr. Top. Microbiol. Immunol. 340, 155-169

22. Yang, J. and Reth, M. (2010) Oligomeric organization of the B-cell antigen receptor on resting cells. Nature 467, 465-469

23. Kläsener, K. et al. (2014) B cell activation involves nanoscale receptor reorganizations and inside-out signaling by Syk. Elife 3, e02069

24. Maecker, H.T. and Levy, S. (1997) Normal lymphocyte development but delayed humoral immune response in CD81-null mice. J. Exp. Med. 185, 1505-1510

25. Mattila, P.K. et al. (2013) The actin and tetraspanin networks organize receptor nanoclusters to regulate $\mathrm{B}$ cell receptor-mediated signaling. Immunity 38, 461-474

26. Rickert, R.C. (2013) New insights into pre-BCR and BCR signalling with relevance to B cell malignancies. Nat. Rev. Immunol. 13, 578-591

27. Sander, S. et al. (2012) Synergy between PI3K signaling and MYC in Burkitt lymphomagenesis. Cancer Cell 22, 167-179

28. Young, R.M. et al. (2015) Survival of human lymphoma cells requires B-cell receptor engagement by self-antigens. Proc. Nat/. Acad. Sci. U. S. A. 112, 13447-13454 


\section{Trends in Cancer}

29. Lenz, G. et al. (2008) Oncogenic CARD11 mutations in human diffuse large B cell lymphoma. Science 319, 1676-1679

30. Sachen, K.L. et al. (2012) Self-antigen recognition by follicular lymphoma B-cell receptors. Blood 120, 4181-4190

31. Cha, S. et al. (2013) Nonstereotyped lymphoma B cell receptors recognize vimentin as a shared autoantigen. J. Immunol. 190, 4887-4898

32. Radcliffe, C.M. et al. (2007) Human follicular lymphoma cells contain oligomannose glycans in the antigen-binding site of the B-cell receptor. J. Biol. Chem. 282, 7405-7415

33. Coelho, V. et al. (2010) Glycosylation of surface Ig creates a functional bridge between human follicular lymphoma and microenvironmental lectins. Proc. Natl. Acad. Sci. U. S. A. 107, 1858718592

34. Linley, A. et al. (2015) Lectin binding to surface Ig variable regions provides a universal persistent activating signal for follicular lymphoma cells. Blood 126, 1902-1910

35. Amin, R. et al. (2015) DC-SIGN-expressing macrophages trigger activation of mannosylated IgM B-cell receptor in follicular lymphoma. Blood 126, 1911-1920

36. Meeker, T. et al. (1985) A clinical trial of anti-idiotype therapy for B cell malignancy. Blood 65, 1349-1363

37. Torchia, J. et al. (2016) Targeting lymphoma with precision using semisynthetic anti-idiotype peptibodies. Proc. Natl. Acad. Sci. U. S. A. $113,5376-5381$

38. Palanca-Wessels, M.C.A. (2015) Safety and activity of the antiCD79B antibody-drug conjugate polatuzumab vedotin in relapsed or refractory B-cell non-Hodgkin lymphoma and chronic lymphocytic leukaemia: a phase 1 study. Lancet Oncol. 16, $704-$ 715

39. Shank, B.R. et al. (2017) Chimeric antigen receptor T cells in hematologic malignancies. Pharmacotherapy 37, 334-345

40. Goebeler, M.-E. and Bargou, R. (2016) Blinatumomab: a CD19/ CD3 bispecific T cell engager (BiTE) with unique anti-tumor efficacy. Leuk. Lymphoma 57, 1021-1032

41. Couzin-Frankel, J. (2013) Breakthrough of the year 2013. Cancer immunotherapy. Science 342, 1432-1433

42. Goodman, A. et al. (2017) PD-1-PD-L1 immune-checkpoint blockade in B-cell lymphomas. Nat. Rev. Clin. Oncol. 14, 203220

43. Hoos, A. (2016) Development of immuno-oncology drugs - from CTLA4 to PD1 to the next generations. Nat. Rev. Drug Discov. 15, 235-247

44. Younes, A. et al. (2016) Nivolumab for classical Hodgkin's lymphoma after failure of both autologous stem-cell transplantation and brentuximab vedotin: a multicentre, multicohort, single-arm phase 2 trial. Lancet Oncol. 17, 1283-1294

45. Armand, P. et al. (2013) Disabling immune tolerance by programmed death-1 blockade with pidilizumab after autologous hematopoietic stem-cell transplantation for diffuse large b-cell lymphoma: results of an international phase II trial. J. Clin. Oncol. 31, 4199-4206

46. Lesokhin, A.M. et al. (2016) Nivolumab in patients with relapsed or refractory hematologic malignancy: preliminary results of a phase Ib study. J. Clin. Oncol. 34, 2698-2704

47. Andorsky, D.J. et al. (2011) Programmed death ligand 1 is expressed by non-Hodgkin lymphomas and inhibits the activity of tumor-associated T cells. Clin. Cancer Res. 17, 4232-4244

48. Menter, T. et al. (2016) Evaluation of the diagnostic and prognostic value of PDL1 expression in Hodgkin and B-cell lymphomas. Hum. Pathol. 54, 17-24

49. Chen, B.J. et al. (2013) PD-L1 expression is characteristic of a subset of aggressive B-cell lymphomas and virus-associated malignancies. Clin. Cancer Res. 19, 3462-3473

50. Myklebust, J.H. et al. (2013) High PD-1 expression and suppressed cytokine signaling distinguish $\mathrm{T}$ cells infiltrating follicular lymphoma tumors from peripheral T cells. Blood 121, 1367-1376

51. Westin, J.R. et al. (2014) Safety and activity of PD1 blockade by pidilizumab in combination with rituximab in patients with relapsed follicular lymphoma: a single group, open-label, phase 2 trial. Lancet Oncol. 15, 69-77
52. Tsirigotis, P. et al. (2016) Programmed death-1 immune check point blockade in the treatment of hematological malignancies. Ann. Med. 3890, 1-12

53. McClanahan, F. et al. (2016) Catching up with solid tumor oncology: what is the evidence for a prognostic role of programmed cel death-ligand 1/programmed cell death-1 expression in B-cell lymphomas? Haematologica 101, 1144-1158

54. van Spriel, A.B. et al. (2009) The tetraspanin protein CD37 regulates IgA responses and anti-fungal immunity. PLoS Pathog. 5 , e1000338

55. de Winde, C.M. et al. (2015) Multispectral imaging reveals the tissue distribution of tetraspanins in human lymphoid organs. Histochem. Cell Biol. 144, 133-146

56. de Winde, C.M. et al. (2016) Tetraspanin CD37 protects agains the development of B cell Iymphoma. J. Clin. Invest. 126, 653666

57. Xu-Monette, Z.Y. et al. (2016) Assessment of CD37 B-cell antigen and cell-of-origin significantly improves risk prediction in diffuse large B-cell lymphoma. Blood 128, 3083-3101

58. Barrena, S. et al. (2005) Aberrant expression of tetraspanin molecules in B-cell chronic lymphoproliferative disorders and its correlation with normal B-cell maturation. Leukemia 19, 13761383

59. Press, O.W. et al. (1989) Treatment of refractory non-Hodgkin's lymphoma with radiolabeled MB-1 (anti-CD37) antibody. J. Clin. Oncol. 7, 1027-1038

60. Zhao, X. et al. (2007) Targeting CD37-positive lymphoid malig nancies with a novel engineered small modular immunopharmaceutical. Blood 110, 2569-2577

61. Beckwith a, K. et al. (2015) Tetraspanins as therapeutic targets in hematological malignancy: a concise review. Front. Physiol. 6, 113

62. Robak, T. et al. (2016) Antibody therapy alone and in combination with targeted drugs in chronic lymphocytic leukemia. Semin. Oncol. 43, 280-290

63. Lapalombella, R. et al. (2012) Tetraspanin CD37 directly mediates transduction of survival and apoptotic signals. Cancer Cell 21 694-708

64. Deckert, J. et al. (2013) A novel anti-CD37 antibody-drug conjugate with multiple anti-tumor mechanisms for the treatment of $\mathrm{B}$ cell malignancies. Blood 122, 3500-3510

65. Dahle, J. et al. (2013) Evaluating antigen targeting and anti-tumo activity of a new anti-CD37 radioimmunoconjugate against nonHodgkin's lymphoma. Anticancer Res. 33, 85-95

66. van Spriel, A.B. et al. (2012) The tetraspanin CD37 orchestrates the alpha beta $_{1}$ integrin-Akt signaling axis and supports longlived plasma cell survival. Sci. Signal. 5, ra82

67. Burger, R. (2013) Impact of interleukin-6 in hematological malig nancies. Transfus. Med. Hemother. 40, 336-343

68. Ferrario, A. et al. (2017) Siltuximab and hematologic malignancies. A focus in non Hodgkin lymphoma. Expert Opin. Investig. Drugs 26, 367-373

69. Szöllósi, J. et al. (1996) Supramolecular complexes of MHC class I, MHC class II, CD20, and tetraspan molecules (CD53, CD81, and CD82) at the surface of a B cell line JY. J. Immunol. 157 , 2939-2946

70. Petrie, R.J. and Deans, J.P. (2002) Colocalization of the B cell receptor and CD20 followed by activation-dependent dissociation in distinct lipid rafts. J. Immunol. 169, 2886-2891

71. Polyak, M.J. et al. (2008) CD20 homo-oligomers physically associate with the $\mathrm{B}$ cell antigen receptor: dissociation upon recepto engagement and recruitment of phosphoproteins and calmodulin-binding proteins. J. Biol. Chem. 283, 18545-18552

72. Zuidscherwoude, M. (2015) The tetraspanin web revisited by super-resolution microscopy. Sci. Rep. 5, 12201

73. Hryniewicz-Jankowska, A. (2014) Membrane rafts as a nove target in cancer therapy. Biochim. Biophys. Acta 1845, 155-165

74. Delos Santos, R.C. (2015) Charming neighborhoods on the cell surface: plasma membrane microdomains regulate receptor tyrosine kinase signaling. Cell Signal. 27, 1963-1976 


\section{Trends in Cancer}

75. Zuidscherwoude, M. (2014) Microdomains in the membrane landscape shape antigen-presenting cell function. J. Leukoc. Biol. 95, 251-263

76. Brown, D.A. and Rose, J.K. (1992) Sorting of GPI-anchored proteins to glycolipid-enriched membrane subdomains during transport to the apical cell surface. Cell $68,533-544$

77. Simons, K. and Ikonen, E. (1997) Functional rafts in cell membranes. Nature 387, 569-572

78. Hemler, M.E. (2005) Tetraspanin functions and associated microdomains. Nat. Rev. Mol. Cell Biol. 6, 801-811

79. Levy, S. and Shoham, T. (2005) The tetraspanin web modulates immune-signalling complexes. Nat. Rev. Immunol. 5, 136-148

80. Charrin, S. et al. (2009) Lateral organization of membrane proteins: tetraspanins spin their web. Biochem. J. 420, 133-154

81. Claas, C. et al. (2001) Evaluation of prototype transmembrane 4 superfamily protein complexes and their relation to lipid rafts. J. Biol. Chem. 276, 7974-7984
82. Hemler, M.E. (2003) Tetraspanin proteins mediate cellular penetration, invasion, and fusion events and define a novel type of membrane microdomain. Annu. Rev. Cell Dev. Biol. 19, 397-422

83. Huang, S. et al. (2005) The phylogenetic analysis of tetraspanins projects the evolution of cell-cell interactions from unicellular to multicellular organisms. Genomics 86, 674-684

84. Wright, M.D. and Tomlison, M.G. (1994) The ins and outs of the transmembrane 4 superfamily. Immunol. Today 15, 588-594

85. S.J. van Deventer, et al., Molecular interactions shaping the tetraspanin web. Biochem. Soc. Trans.(in press).

86. Wright, M.D. et al. (2000) The L6 membrane proteins - a new four-transmembrane superfamily. Protein Sci 9, 1594-1600

87. Hemler, M.E. (2014) Tetraspanin proteins promote multiple cancer stages. Nat. Rev. Cancer 14, 49-60 